\title{
LCLS-TN-09-1
}

\section{Magnetic Measurement Results of the LCLS Undulator Quadrupoles}

\author{
Scott Anderson, Keith Caban, Heinz-Dieter Nuhn, Ed Reese, Zachary Wolf
}

SLAC National Accelerator Laboratory

January 13, 2010

\begin{abstract}
This note details the magnetic measurements and the magnetic center fiducializations that were performed on all of the thirty-six LCLS undulator quadrupoles. Temperature rise, standardization reproducibility, vacuum chamber effects and magnetic center reproducibility measurements are also presented.
\end{abstract}

\section{Introduction ${ }^{1}$}

The Linac Coherent Light Source (LCLS) undulator beam line has 33 girders, each with a LCLS undulator quadrupole which focuses and steers the beam through the beam line. Each quadrupole has main quadrupole coils, as well as separate horizontal and vertical trim coils. Thirty-six quadrupoles, thirty-three installed and three spares were, manufactured for the LCLS undulator system and all were measured to confirm that they met requirement specifications for integrated gradient, harmonics and for magnetic center shifts after current changes. ${ }^{2}$ The horizontal and vertical dipole trims of each quadrupole were similarly characterized. Each quadrupole was also fiducialized to its magnetic center. All characterizing measurements on the undulator quads were performed with their mirror plates on and after a standardization of three cycles from -6 to +6 to -6 amps. Since the undulator quadrupoles could be used as a focusing or defocusing magnet depending on their location, all quadrupoles were characterized as focusing and as defocusing quadrupoles.

A subset of the undulator quadrupoles were used to verify that the undulator quadrupole design met specifications for temperature rise, standardization reproducibility and magnetic center reproducibility after splitting. The effects of the mirror plates on the undulator quadrupoles were also measured.

\section{Integrated Gradient and Harmonics Measurements}

All thirty-six quadrupoles had their integrated gradients measured at currents between -6 and +6 amps (up ramp) and from +6 to -6 amps (down ramp) in 0.5 amp steps. The measured values given throughout this paper were made on the up ramp of the current cycle. The nominal integrated gradient for the undulator quadrupoles is +3.0 Tesla (focusing quadrupole) or -3.0 Tesla (defocusing quadrupole), which roughly corresponds to main coil currents of +4.3 and -4.3 amps respectively. The average integrated gradient at the nominal currents of +4.3 and -4.3 amps, are $3.0233+/-0.0161$ and $-3.0531+/-0.0161$ Tesla respectively. Figure 1 shows the integrated gradient at +4.3 amps for all of the undulator quadrupoles.

\footnotetext{
${ }^{1}$ Work supported in part by the DOE Contract DE-AC02-76SF00515. This work was performed in support of the LCLS project at SLAC.

${ }^{2}$ Heinz-Dieter Nuhn, LCLS Physics Document 1.4-001 "General Undulator System Requirements”, Rev 4
} 


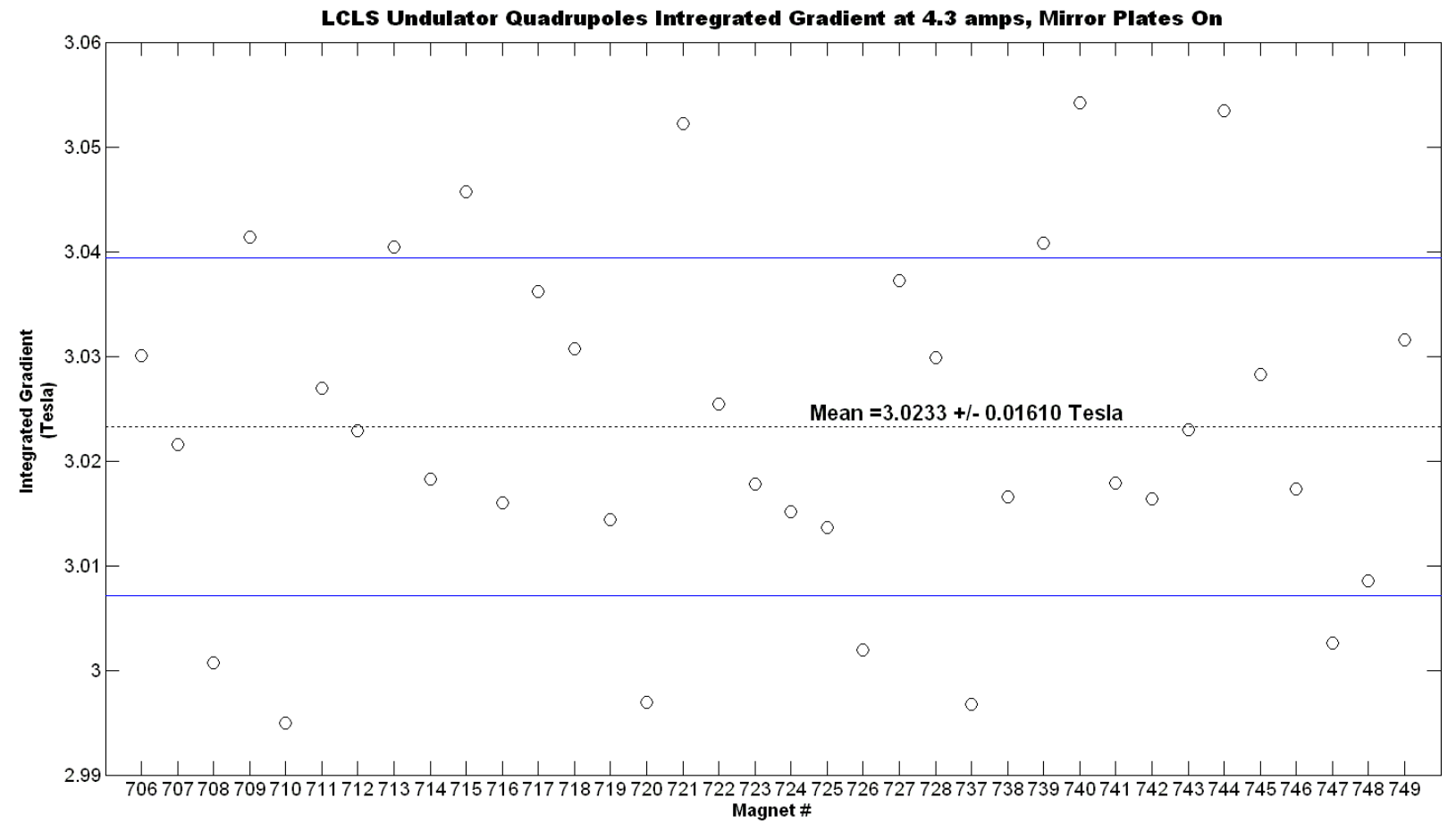

Figure 1. Integrated Gradient at +4.3 amps (calculated using 3 rd order fit of data from -6 to +6 amps in 0.5 amps steps). Each of the thirty-six undulator quadrupoles was assigned a barcode number as its name, for example quadrupole 000706 is the quadrupole listed on this graph as 706 .

The harmonics for all of the quadrupoles were measured at the maximum current values of 6 amps and at the nominal current values. The harmonics for the all the undulator quads were acceptable. Table 1 lists the maximum and mean normalized harmonics for harmonic numbers $\mathrm{n}$ $=3,4,6$ and 10 for all the undulator quads at the nominal currents. A summary plot of these harmonics at $-4.3 \mathrm{amps}$, for all the magnets, is in figure 2 . The plot for +4.3 amps is nearly identical.

\begin{tabular}{|c|c|c|c|}
\hline Harmonic \# & Maximum & Mean & Standard Deviation \\
\hline 3 & $7.642 \mathrm{E}-03$ & $4.042 \mathrm{E}-03$ & $1.5960 \mathrm{E}-03$ \\
\hline 4 & $2.547 \mathrm{E}-03$ & $9.994 \mathrm{E}-04$ & $4.5262 \mathrm{E}-04$ \\
\hline 6 & $2.441 \mathrm{E}-03$ & $2.184 \mathrm{E}-03$ & $1.5964 \mathrm{E}-04$ \\
\hline 10 & $7.852 \mathrm{E}-04$ & $2.565 \mathrm{E}-04$ & $1.0950 \mathrm{E}-04$ \\
\hline
\end{tabular}

Table 1: Normalized (normalized to the fundamental harmonic $n=2$ ) Harmonics: $n=3$ (sextupole), 4 (octupole), 6 and 10 maximum, mean and standard deviation at $+/-4.3$ amps. Harmonics given at radius $=0.004133$ meters . 


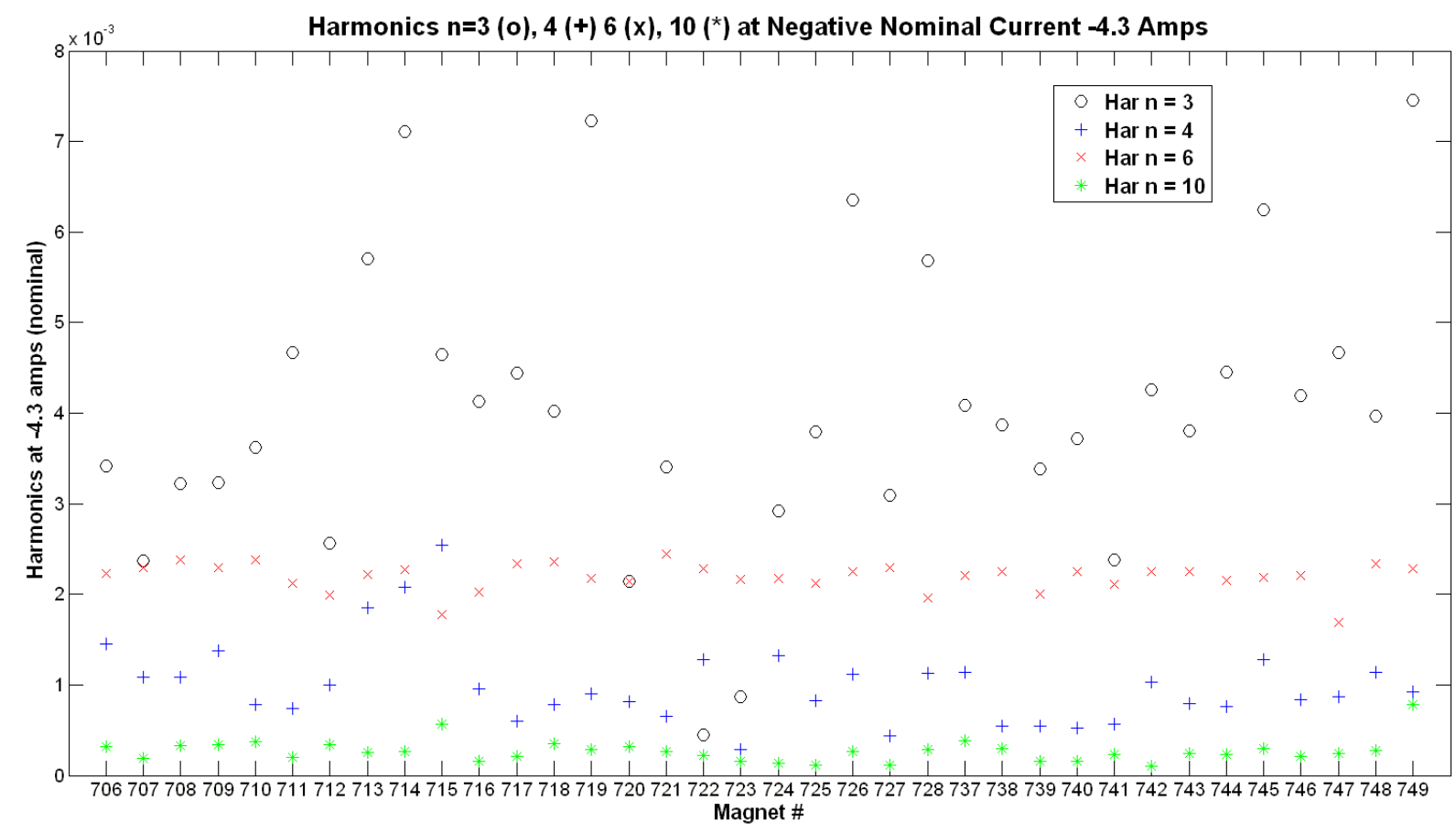

Figure 2: Normalized harmonics $(\mathrm{n}=3,4,6$ and 10) for all quadrupoles at -4.3 amps. Harmonics given at radius $=0.004133$ meters.

\section{Horizontal and Vertical Trim Measurements}

Each quadrupole has separately wound and powered horizontal and vertical trim coils. Each trim's characteristics were measured using the same rotating coil used for the main coil measurements. The trims were measured in a $+/-0.5 \mathrm{amp}$ square pattern (values are listed in Table 2), with the main coils set at both of the two nominal currents. Each horizontal trim gave on average an integrated field of $5.33+/-0.06 \times 10^{-4} \mathrm{Tm}$ for each $1 \mathrm{amp}$ delta change which is equivalent to a quadrupole displacement of $175.5+/-2.1 \mu \mathrm{m}$. Each vertical trim gave on average an integrated field of $5.20+/-0.08 \times 10^{-4} \mathrm{Tm}$ for each $1 \mathrm{amp}$ delta change which is equivalent to a quadrupole displacement of $171.6+/-2.5 \mu \mathrm{m}$. The average magnetic center change, due to hysteresis, between the start of the $+/-0.5 \mathrm{amp}$ current square pattern and the end for all the quadrupoles was $0.77+/-0.53 \mu \mathrm{m}$ in the horizontal and $0.96+/-0.54 \mu \mathrm{m}$ in the vertical.

After the undulator quadrupoles were installed the trims were used to correct the beam when the quadrupole was offset by a known amount. There should be a clear correlation between the quadrupole offset, which causes a dipole field kick related to the quadrupole strength and the trim field strength to counter it. It was noticed that the trims over corrected by about $15 \%$. The trims of a spare undulator quadrupole were remeasured using a stretched wire system and it was found that the dipole calibration of the DC1B rotating coil used in the original measurements was low by $15.7 \%$. The quadrupole calibration of the DC1B rotating coil was also checked and found to be the same as before. Since the DC1B rotating coil was used to measure the dipole field of the trims, their measured dipole transfer functions (integrated field per ampere of current) were $15.7 \%$ low. To correct this measurement error, all of the undulator quadrupole 
trims will have their transfer function coefficients divided by 0.8642 which will fully correct for the dipole coil calibration error. Appendix A gives a more thorough explanation of coil recalibration process.

Using the above correction, each horizontal trim gave, on average, an integrated field of 6.17 $+/-0.07 \times 10^{-4} \mathrm{Tm}$ for each $1 \mathrm{amp}$ delta change which is equivalent to a quadrupole displacement of $203.1+/-2.4 \mu \mathrm{m}$. Each vertical trim gave on average an integrated field of $6.02+/-0.09 \times 10^{-4}$ Tm for each $1 \mathrm{amp}$ delta change which is equivalent to a quadrupole displacement of $198.6+/-$ $2.5 \mu \mathrm{m}$. The average magnetic center change, due to hysteresis, after the $+/-0.5 \mathrm{amp}$ current square pattern measurements for all the quadrupoles was $0.89+/-0.61 \mu \mathrm{m}$ in the horizontal and $1.11+/-0.62 \mu \mathrm{m}$ in the vertical. All subsequent dipole field related measurements take into account the DC1B rotating coil dipole recalibration.

\begin{tabular}{|c|c|c|}
\hline Position Description & H-Cur & V-Cur \\
\hline Center & $0 \mathrm{~A}$ & $0 \mathrm{~A}$ \\
\hline Upper Right Corner & $+0.5 \mathrm{~A}$ & $+0.5 \mathrm{~A}$ \\
\hline Lower Right Corner & $+0.5 \mathrm{~A}$ & $-0.5 \mathrm{~A}$ \\
\hline Lower Left Corner & $-0.5 \mathrm{~A}$ & $-0.5 \mathrm{~A}$ \\
\hline Upper Left Corner & $-0.5 \mathrm{~A}$ & $+0.5 \mathrm{~A}$ \\
\hline Center & $0 \mathrm{~A}$ & $0 \mathrm{~A}$ \\
\hline
\end{tabular}

Table 2: Current sequence for trim square pattern

\section{Center Shifts from +/- 20\% Gradient Change}

During the Beam Based Alignment (BBA) procedure each undulator quadrupole has its main coil current (and its gradient) changed by $+/-20 \%$ from its nominal value. The magnetic center of each quadrupole is required to move less than $+/-3 \mu \mathrm{m}$ during that change. The center change was measured around both nominal currents, by increasing the current by 1 amp, bringing it back to nominal, lowering the current by 1 amp and bringing it back to nominal current. The BBA center change measurements were done in $0.5 \mathrm{amp}$ steps with magnetic center measurements taken at each step. All the quadrupoles had measured center shifts below $3 \mu \mathrm{m}$ for the nominal to $+20 \%$ and the nominal to $-20 \%$ gradient changes. The mean center shifts of all the quadrupoles are listed in table 3 and show that on average the center shifts are $1 \mu \mathrm{m}$ or less.

\begin{tabular}{|c|c|c|}
\hline Axis & Center shift for +1 amp from nominal & Center shift for -1 amp from nominal \\
\hline $\mathrm{X}$ & $0.89+/-0.61 \mu \mathrm{m}$ & $1.04+/-0.73 \mu \mathrm{m}$ \\
\hline $\mathrm{Y}$ & $0.64+/-0.47 \mu \mathrm{m}$ & $0.84+/-0.78 \mu \mathrm{m}$ \\
\hline
\end{tabular}

Table 3: Magnetic Center Shift after $+/-1$ amp change on quad main. 


\section{Magnetic Fiducialization}

Fiducializing a quadrupole relates its outside tooling sockets to its center point in $\mathrm{x}, \mathrm{y}$ and $\mathrm{z}$, and to its rotational planes of pitch, roll and yaw. Each undulator quadrupole was fiducialized to its magnetic centerline using a vibrating wire fiducialization system. ${ }^{3}$ Using the vibrating wire fiducial system allows positioning of the quadrupoles magnetic centerline onto the girder centerline to a higher accuracy than if they were placed using only mechanical fiducialization. The undulator quadrupole fiducialization process and its errors are described below.

To fiducialize a quadrupole using the vibrating wire system, it is first mounted on a cam mover system which can move each quadrupole independently in the $\mathrm{x}, \mathrm{y}$, pitch and yaw. The quadrupole then has a wire stretched through it which is connected to a function generator that can output a sine wave signal at the $2^{\text {nd }}$ harmonic of the wire mechanical fundamental frequency, for alignment in $\mathrm{x}$ and $\mathrm{y}$, and at the $4^{\text {th }}$ harmonic, for alignment in pitch and yaw. The $2^{\text {nd }}$ and $4^{\text {th }}$ harmonics are used to cancel the effect of the earth's magnetic field on the vibration measurements. When the function generator outputs one of these frequencies, the wire vibrates if it is not located on the magnetic centerline of the quadrupole. To locate the wire on the magnetic centerline, each quadrupole is scanned, using the cam mover system, in $\mathrm{x}, \mathrm{y}$, pitch and yaw and set to the zero vibration point for each of these directions. When the wire is at the zero vibration points for $\mathrm{x}, \mathrm{y}$, pitch and yaw, it is on the magnetic centerline of the quadrupole and its location can be related to the quadrupole tooling sockets using a coordinate measuring machine (CMM), on which the whole vibrating wire system rests. The CMM locates the 5 tooling sockets of each undulator quadrupole and relates them to the position of the wire, thus fiducializing the quadrupole to its magnetic axis. The wire location is determined using four wire position detectors, two for the horizontal wire position and two for the vertical, with one of each detector on either side of the magnet. Each wire position detector is a laser/slit/photodiode system set midway in a u-shaped holder with two tooling balls on its end cradled in a mount that is attached to a movable stage. To find the wire, each wire position detector moves until the wire interrupts half of the laser beam shining onto a slit/photodiode detector, then the two tooling balls that are located by the CMM. The distance from the tooling balls to the wire is known because that distance from the tooling balls to the point where half the laser beam is interrupted is calibrated beforehand. With the wire and the undulator quadrupole tooling sockets located, to the undulator quadrupole is fiducialized to its magnetic centerline.

While the $\mathrm{x}, \mathrm{y}$, pitch and yaw directions of the undulator quadrupoles were located using the vibrating wire fiducial system, the roll and $\mathrm{z}$ directions were not. Roll and $\mathrm{z}$ were located using the quadrupoles mechanical characteristics, such as its pole tips and its side of its laminations. Each quadrupole was fully mechanically fiducialized and this mechanical fiducial data was used to determine its roll and its $\mathrm{z}$ center. The roll and $\mathrm{z}$ data was integrated with the wire fiducial data to give a complete fiducialization of each quadrupole. The $\mathrm{x}, \mathrm{y}$, pitch and yaw mechanical fiducial data was also used as a cross check of the magnetic centerline fiducial values.

The undulator quadrupole had to be set on the girder to tolerances of $25 \mu \mathrm{m}$ in $\mathrm{x}$ and $\mathrm{y}, 0.5$ $\mathrm{mm}$ in $\mathrm{z}$ and to 15,15 and $20 \mathrm{mrad}$ for pitch, yaw and roll respectively. The vibrating wire fiducialization errors come from the measurement steps needed to align the quadrupole to the

${ }^{3}$ Z. Wolf, "A Vibrating Wire System For Quadrupole Fiducialization", LCLS-TN-05-11, May, 2005. 
wire and from locating the wire and the quadrupole in $\mathrm{x}, \mathrm{y}$, pitch and yaw. The roll and $\mathrm{z}$ fiducial errors are determined by the measurement of the mechanical surfaces of the magnet.

There are four sources of uncertainties for the wire fiducial system. The first is the uncertainty when calibrating the distance from the wire detector tooling balls to point where the laser/slit/photodiode system is half shadowed by the wire. The second is the uncertainty in locating the positions of the detector tooling balls after they have located the wire. The third is the uncertainty in placing the wire on the magnetic centerline of each undulator quadrupole. The fourth is the uncertainty in locating the tooling sockets of the undulator quadrupoles. The total uncertainty is the sum in quadrupole of the above uncertainties. The roll and $\mathrm{z}$ fiducialization uncertainties come from the mechanical fiducialization measurements errors and the uncertainty in reproducing the mechanical fiducialization on the wire fiducial system CMM. Table 4 lists the average fiducialization uncertainties for all the undulator quadrupoles and their sum in quadrature values.

\begin{tabular}{|c|c|c|c|c|c|c|}
\hline Uncertainty & $\begin{array}{c}\mathrm{X} \\
(\mu \mathrm{m})\end{array}$ & $\begin{array}{c}\mathrm{Y} \\
(\mu \mathrm{m})\end{array}$ & $\begin{array}{c}\text { Pitch } \\
(\mathrm{mrad})\end{array}$ & $\begin{array}{c}\text { Yaw } \\
(\mathrm{mrad})\end{array}$ & $\begin{array}{c}\mathrm{Z} \\
(\mu \mathrm{m})\end{array}$ & $\begin{array}{c}\text { Roll } \\
(\mathrm{mrad})\end{array}$ \\
\hline Wire Detector Calibration & 1.21 & 1.17 & 0.004 & 0.003 & ---- & ---- \\
\hline Wire Detector Tooling Balls & 2.77 & 3.16 & 0.010 & 0.007 & ---- & ---- \\
\hline Quad Positioning & 1.99 & 0.55 & 0.021 & 0.369 & 3.68 & 0.141 \\
\hline Quad Tooling Sockets & 1.84 & 3.44 & 0.046 & 0.024 & 26.18 & 0.324 \\
\hline Total Uncertainty & $\mathbf{4 . 0 6}$ & $\mathbf{4 . 8 5}$ & $\mathbf{0 . 0 5 2}$ & $\mathbf{0 . 3 6 9}$ & $\mathbf{2 6 . 4 4}$ & $\mathbf{0 . 3 5 3}$ \\
\hline
\end{tabular}

Table 4: Vibrating Wire System and Mechanical Fiducial Uncertainties.

These average uncertainties were determined for $\mathrm{x}, \mathrm{y}$, pitch and yaw in the following ways. The wire detector calibration uncertainty is the sum in quadrature of the standard deviations of the two $\mathrm{x}$ wire detector's tooling ball to laser/slit/photodiode distances measured using the detector flip technique ${ }^{3}$. The wire detector tooling ball uncertainty is the standard deviation of the CMM measurement fits of the wire detectors tooling balls. The uncertainty in the quadrupole positioning is the rms offset between the wire and each quadruples' magnetic centerline as measured by the residual vibration that the wire had after the quadruples' placement by the cam mover system. The uncertainty in locating the quadrupole tooling sockets is the standard deviation of the CMM measurement fits of the tooling sockets. The $\mathrm{z}$ and roll positioning uncertainties are of the tooling sockets during the positioning of the quadrupoles on the vibrating wire system apparatus. The quadrupole tooling sockets uncertainties for $\mathrm{z}$ and roll are the standard deviations of the multiple mechanical fiducial measurements of an undulator quadrupole.

The total fiducial uncertainties for the $\mathrm{x}$ and $\mathrm{y}$ are below the tolerance of $10 \mu \mathrm{m}$ given for the undulator quadrupoles fiducialization process. ${ }^{2}$ The pitch, yaw and roll errors are well below the respective 15, 15 and $20 \mathrm{mrad}$ tolerances that the undulator quads have for their alignment on the girders. The $\mathrm{z}$ error is also well below the $0.5 \mathrm{~mm}$ longitudinal positioning tolerance.

The undulator quadrupole fiducial stability tolerance for $\mathrm{x}$ and $\mathrm{y}$ is $25 \mu \mathrm{m}$. The above errors indicate that this tolerance should be met. As a check, four of the undulator quadrupoles were 
fiducialized two or more times. The mean difference between the first and the subsequent fiducial measurements for these quadrupoles are $3.7 \mu \mathrm{m}$ in the $\mathrm{x}$ and $1.1 \mu \mathrm{m}$ in the $\mathrm{y}$.

Another way to verify that the $\mathrm{x}$ and $\mathrm{y}$ magnetic fiducial values are reasonable is to compare them to the mechanical fiducial values. The comparison shows that the magnetic centers of the quadrupoles are on average within $16 \mu \mathrm{m}$ of their mechanical centers in the horizontal and vertical axes. To understand if this offset is reasonable, the magnetic to mechanical center offsets for the undulator quadrupoles can be calculated using the mechanical fiducial data and first order perturbation equations. ${ }^{4}$ For the undulator quadrupoles, the magnetic to mechanical offsets calculated using the mechanical fiducial data and perturbation equations are $14 \mu \mathrm{m}$ and $16 \mu \mathrm{m} \mathrm{rms}$ in the horizontal and vertical axes respectively. Figure 3 shows the magnetic center and the mechanical center differences for all the undulator quadrupoles.
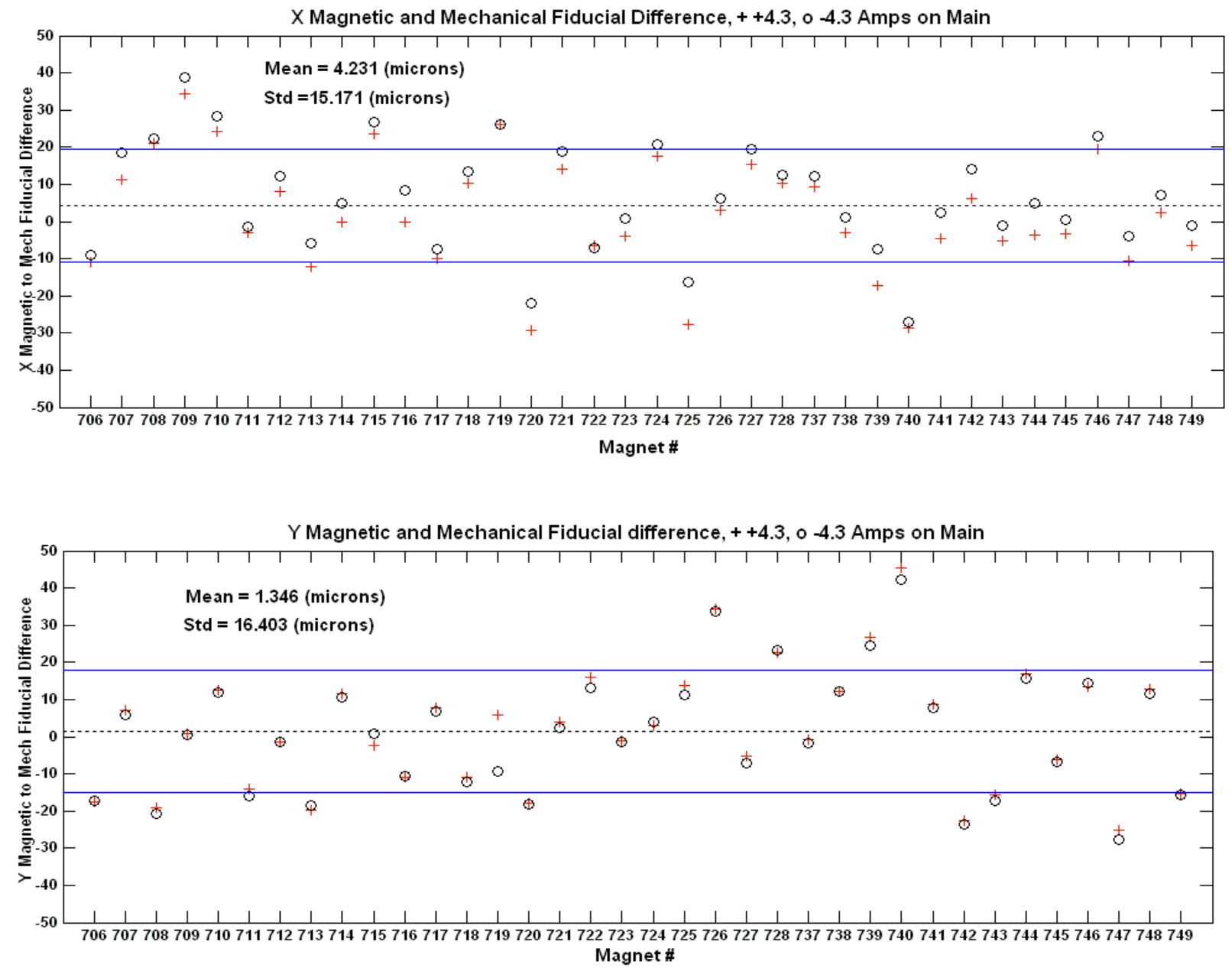

Figure 3: Magnetic and Mechanical Fiducial Center Differences.

4 K. Halbach, "First order perturbation effects in iron dominated two dimensional symmetrical multipoles," Nuclear Instruments and Methods, Vol. 74, pp 147-164, 1969. 


\section{Effects of Magnet Split and Vacuum Chamber on Magnetic Measurements}

All undulator quadrupoles were magnetically measured and fiducialized to their magnetic centers without their vacuum chamber installed, so it is of interest to understand what effect splitting a quadrupole and installing a vacuum chamber has on these measurements.

One quadrupole's integrated gradient was measured with and without a vacuum chamber installed. The ratio of the integrated gradient without the vacuum chamber installed to with it installed was $1.0017+/-0.0024$, which is less than the $1 \%$ absolute accuracy demanded for the value of the integrated gradient.

The effect of splitting and reassembling a quadrupole on its magnetic center was measured. First, the magnetic center was measured, then the quadrupole was carefully split and reassembled using the standard LCLS written procedure and then the magnetic center was measured again. The magnetic center moved horizontally by $1.3+/-0.8 \mu \mathrm{m}$ and vertically by $1.4+/-0.1 \mu \mathrm{m}$.

The effect on the quadrupole's fiducialization from splitting and reassembling and from splitting, inserting a vacuum chamber and reassembling were also studied. Four separate wire fiducial data sets were made on one of the undulator quadrupoles, with each set consisting of fiducializing the quadrupole at +4.3 and -4.3 amps. The first two sets were control sets (Baseline 1 and Baseline 2), the third set (Split) was made after the quadrupole was split and reassembled and the fourth set (Vacuum Chamber) was made after a vacuum chamber was installed in the quadrupole, which also required splitting and reassembly. The splitting and reassembly was done per the LCLS written procedure. The resulting data shows little effect due either to splitting and reassembling the quadrupole or due to installing a vacuum chamber. The fiducialization differences to the first measurement set (Baseline 1) are listed in table 5.

\begin{tabular}{|c|c|c|}
\hline & X fiducial offset & Y fiducial offset \\
\hline Baseline 2 & $1.1+/-3.7 \mu \mathrm{m}$ & $0.5+/-3.6 \mu \mathrm{m}$ \\
\hline Split & $-0.4+/-3.7 \mu \mathrm{m}$ & $0.8+/-3.6 \mu \mathrm{m}$ \\
\hline Vacuum Chamber & $-2.3+/-3.7 \mu \mathrm{m}$ & $0.1+/-3.6 \mu \mathrm{m}$ \\
\hline
\end{tabular}

Table 5: Magnet Split and Vacuum Chamber effects using Quadrupole Fiducialization

\section{Temperature Measurements.}

The undulator quadrupoles are solid wire electro-magnets which are operated in the temperature controlled environment of the undulator hall. To understand the temperature rise of a quadrupole, measurements were made at four locations on the magnet assembly: on an upper coil inside the mirror plates, on a lower coil inside the mirror plates, on the laminations outside the mirror plates and on the magnet base plate with the quadrupole main current set to 6 amps (the highest operating current) for 14 hours and at 4.5 amps (4.3 amps is the nominal current) for 10 hours on a single quadrupole. The delta temperatures for these data sets are listed in table 6 and plotted in figure 4. The temperature rise of the core (Outer Lamination) was $8.0^{\circ} \mathrm{C}$ after 14 hours at 6 amps which is less than the specification of a core temperature rise of $9.0^{\circ} \mathrm{C}$ after 4 
hours. All measurements were taken with the mirror plates on and in a temperature controlled room where the ambient temperature was $\sim 21^{\circ} \mathrm{C}$.

\begin{tabular}{|c|c|c|}
\hline Probe Location & $\Delta \mathrm{T}, \mathbf{4 . 5}$ amps for 10 hours & $\Delta \mathrm{T}, \mathbf{6}$ amps for 14 hours \\
\hline Upper Coil $\left({ }^{\circ} \mathbf{C}\right)$ & 8.3 & 13.9 \\
\hline Lower Coil $\left({ }^{\mathbf{0}} \mathbf{C}\right)$ & 6.9 & 12.7 \\
\hline Outer Laminations $\left({ }^{\mathbf{0}} \mathrm{C}\right)$ & 4.6 & 8.0 \\
\hline Base $\left({ }^{\mathbf{0}} \mathbf{C}\right)$ & 1.6 & 3.0 \\
\hline
\end{tabular}

Table 6: Temperature rise measurements at 4.5 and 6.0 amps.

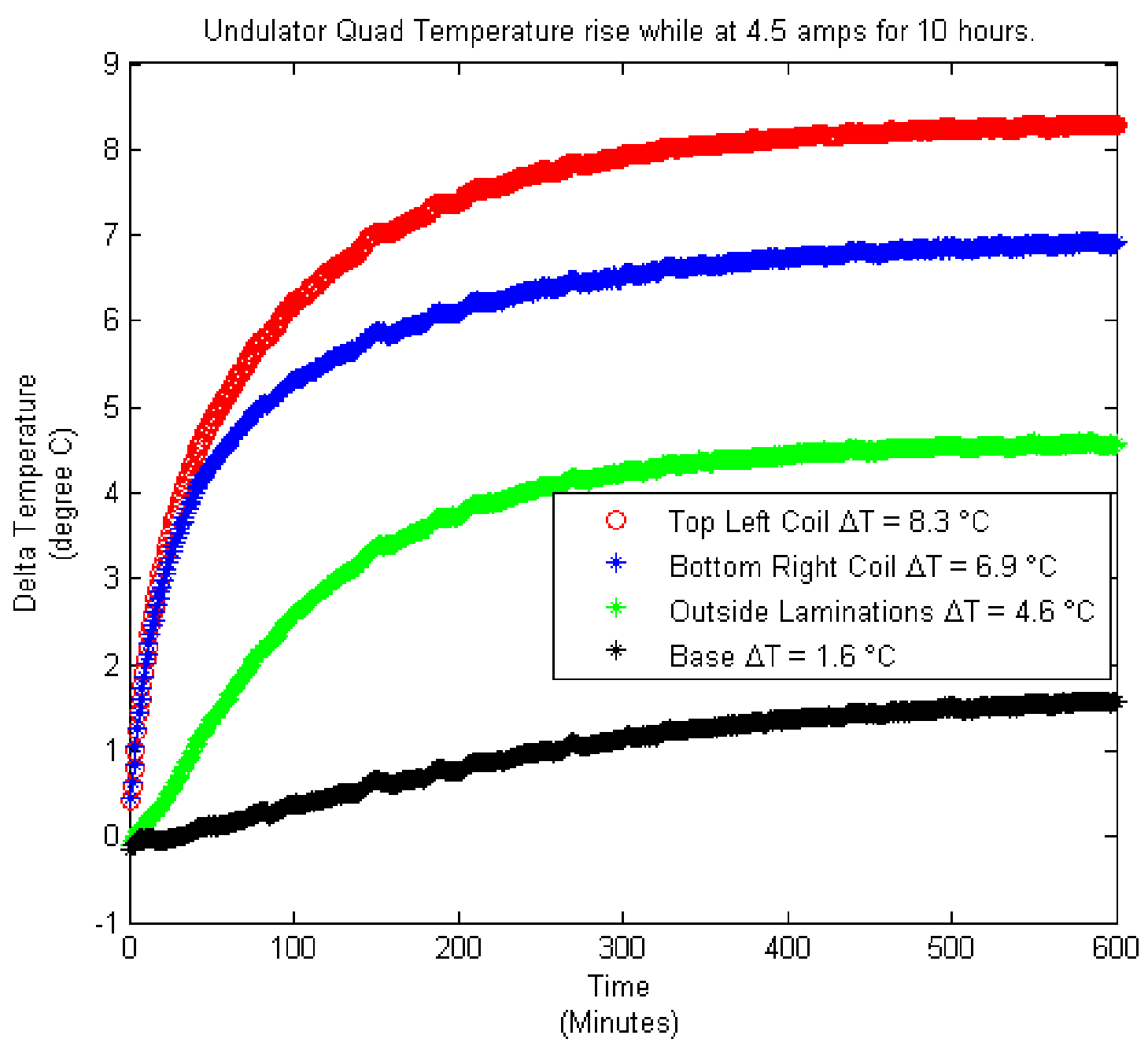

Figure 4: Temperature measurement at 4.5 amps for 10 hours. Mirror plates on. 
Additionally, before the integrated gradient, harmonics, BBA and trim measurements were made, each quad was warmed up for 5 hours and their temperatures were recorded at the beginning and end of the 5 hour period. Temperatures were measured on an upper coil, on an outer lamination and on the magnet base for these measurements. The average delta temperature rise for each location is listed in Table 7.

\begin{tabular}{|c|c|}
\hline Probe Location & Average $\boldsymbol{\Delta}$ T for 5 hour warmup at -4.3 amps \\
\hline Upper Coil $\left({ }^{\circ} \mathbf{C}\right)$ & 7.2 \\
\hline Outer Laminations $\left({ }^{\circ} \mathbf{C}\right)$ & 4.2 \\
\hline Base $\left({ }^{\circ} \mathbf{C}\right)$ & 1.2 \\
\hline
\end{tabular}

Table 7: Average temperature rise of quad after 5 hour warmup.

\section{Standardization Trip Test Measurements.}

Each quadrupole is specified to provide an integrated gradient with a $0.3 \% \mathrm{rms}$ accuracy following its standardize cycles. To verify that the designated three standardize cycles from 6.0 to +6.0 to -6.0 amps were adequate to achieve this, a trip test was performed. The trip test was made by first standardizing a quadrupole and then measuring its integrated gradient twice at +6.0 amps. The magnet's power supply was then tripped off and turned back on again. The quadrupole was then standardized again and its integrated gradient was measured twice at +6.0 amps. The ratio of the integrated gradient measured after the power supply trip to that measured before the trip is is $1.00066+/-0.00029$, which is well below the $0.3 \%$ accuracy needed for the integrated gradient.

\section{Measurements With and Without Mirror Plates.}

The undulator quadrupole has removable mirror plates which were installed on each quadrupole during all of the standard measurements because using the mirror plates lessen the cross talk effects between the quadrupole and the nearby undulator end plate. Never the less, our measurements were made to characterize the effects of having the quadrupole mirror plates installed versus not having them installed and having the undulator end plate at the nominal proximity and it being removed. When the undulator end plate was installed for the tests it was located at the nominal proximity to the undulator quadrupole as it would be on the girder. One quadrupole's integrated gradient, magnetic center and harmonics were characterized in four setups: quadrupole mirror plates on with undulator end plate in nominal proximity, quadrupole mirror plates on with undulator end plate removed, quadrupole mirror plates off with undulator mirror plate in nominal proximity, and quadrupole mirror plates off with an undulator mirror plate removed. The integrated gradient of the quadrupole with the mirror plates installed sees no difference between having the undulator end plate in proximity or removed, within the error of the measurement. The integrated gradient does increase slightly with the undulator mirror in proximity when the quadrupoles mirror plates are removed. Values are listed in table 8. 


\begin{tabular}{|l|c|c|}
\hline & $\begin{array}{c}\text { Quad Mirror On, Ratio Undulator End } \\
\text { Plate Installed/Removed }\end{array}$ & $\begin{array}{c}\text { Quad Mirror Off, Ratio Undulator End } \\
\text { Plate Installed /Removed }\end{array}$ \\
\hline $\begin{array}{l}\text { Integrated } \\
\text { Gradient Ratio }\end{array}$ & $\mathbf{1 . 0 0 0 3}+/ \mathbf{- 0 . 0 0 0 3}$ & $\mathbf{1 . 0 0 1 2 + / - \mathbf { 0 . 0 0 0 3 }}$ \\
\hline
\end{tabular}

Table 8: Effect of Undulator Mirror Plate on Quadrupole, with and without its Mirror Plate

The harmonics values of the quadrupole do not change appreciably whatever the quadrupole mirror plate configuration. Normalized harmonic values are listed in table 9.

\begin{tabular}{|c|l|l|l|l|}
\hline Quad Mirror Plates & \multicolumn{1}{|c|}{ On } & ON & OFF & OFF \\
\hline $\begin{array}{l}\text { Undulator } \\
\text { End Plate }\end{array}$ & Installed & Removed & Installed & Removed \\
\hline Harmonic \# & & & & \\
\hline 3 & 0.47797 & 0.43745 & 0.40069 & 0.49577 \\
\hline 4 & 0.11625 & 0.11468 & 0.12329 & 0.13179 \\
\hline 6 & 0.22455 & 0.22234 & 0.21789 & 0.22602 \\
\hline 10 & 0.03093 & 0.03462 & 0.03509 & 0.22602 \\
\hline
\end{tabular}

Table 9: Normalized harmonics $(n=3,4,6 \& 10)$. Harmonics are normalized to $n=2$ the quadrupole term.

There is also no effect from the undulator end plate on the BBA magnetic center movement measurements, regardless if the quadrupole has its mirror plates on or off.

\section{Conclusion}

All 36 undulator quadrupoles were magnetically characterized with all of the quadrupoles meeting their specified tolerances. These measurements confirmed that the undulator quadrupoles met requirement specifications for integrated gradient, harmonics and for magnetic center shifts after current changes. The horizontal and vertical dipole trims of each quadrupole also met their specifications. Each quadrupole was fiducialized to its magnetic center to less than $5 \mu \mathrm{m}$ in the horizontal and vertical axis. 


\section{Appendix A - Horizontal and Vertical Trim Coil Calibration}

The Horizontal and Vertical trims of the undulator quad have been used to correct the beam when the quadrupole was offset by a known amount. There should be a clear correlation between the quadrupole offset, which causes a dipole field kick related to the quadrupole strength and the trim field strength to counter it. It was noticed that the trims over corrected by about $15 \%$. To determine the cause a spare undulator quadrupole, 000709, was measured again to understand the cause of this problem. It measuring the quadrupole using the stretched wire system is was found that the main quadrupole field was correct, but that the trim fields as measured by the wire were too high by $\sim 14 \%$. To understand how the coil originally measured the trims too high by $\sim 14 \%$, the coil was calibrated in a known dipole. This dipole calibration showed the sensitivity of the DC1B coil was found to be too low by $13.58 \%$. Once the calibration of the DC1B coil constant and dipole sensitivity factor were complete the measurement of $\mathrm{H}$ and $\mathrm{V}$ trims with the new coil constant (CC) and sensitivity factor (SF) were done. The coil constant is a function of the number of turns and the radius of the coils. When the quadrupole coil constant was determined it gave a radius of 0.0041328 meters. To measure the dipole trims properly the coil constant for the dipole only should be determined and a sensitivity factor needs to be determined that relates the strength of the dipole when using the quadrupole radius.

a) The Coil Constants (CC) of DC1B coil were determined using a stretched wire calibration. ECHO7 dipole 3D406 Serial 5 was measured with the wire and then using DC1B coil A and coil B were separately. The horizontal $(\mathrm{H})$ trim was also measured with the stretched wire, but the trim field has a large sextupole component so the 3D4 measurements were used to make the coil calibrations. The coil constants can be compared if one remembers that the double coil is a sum for coils A and B and thus is coil constant is half the average of the A and B coil constants. The coil constants (CC) from the dipole calibration are:

i) Coil A: 5.64737. $1 / 2$ of $\mathrm{CC}$ for Coil A is 2.79975

ii) Coil B: $5.5529 \quad 1 / 2$ of $\mathrm{CC}$ for Coil B is 2.36742

iii) Double Coil: 2.80007. ( $1 / 2$ of $\mathrm{A}$ and B).

b) Knowing the dipole coil constants allows one to calculate the Sensitivity Factor for the coils. The sensitivity factor is needed because the harmonic value of each pole (dipole, quad, sext) is calculated using the following formula:

i) Harmonic Field $=$ (harmonic number $)^{*}($ integrated voltage $) /\left((\# \text { turns })^{*}(\right.$ Coil Radius)*(SF). SF is the sensitivity factor.

ii) The Coil Radius of the wire bundle for DC1B is set to 0.004133 for the quadrupole measurements and it derived by calibrating the coil in quadrupole field measured using the stretched wire. To make the dipole measurements correct using this radius 
a sensitivity factor is used. The sensitivity factor is the ratio of the dipole coil constant determined by the number of turns and the quadrupole coil radius of 0.004133 and the dipole coil constant determined by the calibrating the coil using a dipole field. This ratio for the DC1B coils are:

(1) Coil A: 0.85692, Coil B: 0.87149

(2) Double Coil: 1.72841 ( $1 / 2$ is 0.86421 ) The Double Coil was used to measure the dipole harmonics of the original LCLS quadrupole measurements, so the trim values are low $86.42 \%$ low. All trims, except those on 000709 will be have their coefficients modified by the factor 0.8642 . A text file has been placed in each undulator quads Final Results folder that reads XCU-FACTOR: 0.8642 and YCUFACTOR: 0.8642. This file will stay there until the trims are remeasured. 